\title{
On the Structured Distance to Uncontrollability
}

\author{
Michael Karow* $\quad$ Daniel Kressner ${ }^{\dagger}$
}

September 15, 2008

\begin{abstract}
This article is concerned with the structured distance to uncontrollability of a linear time-invariant system and relates this concept to a variation of the $\mu$-value. The developed framework is applied to derive computational expressions for the class of real perturbations as well as for Hermitian, symmetric, and skew-symmetric perturbations in a relatively simple manner. Examples demonstrate that the structured distance can differ from the standard, unstructured distance to uncontrollability by an arbitrary amount. It is also shown how systems of higher order can be addressed.
\end{abstract}

\section{Introduction}

A matrix pair $(A, B) \in \mathbb{C}^{n \times n} \times \mathbb{C}^{n \times p}$ is said to be controllable if $\operatorname{rank}[A-\lambda I, B]=n$ for all $\lambda \in \mathbb{C}$. By a result of Kalman [11], this coincides with the definition of controllability of the associated linear time-invariant system $\dot{x}=A x+B u$. A reliable way to check controllability numerically is to compute the distance $\delta(A, B)$ of a given matrix pair $(A, B)$ to the nearest uncontrollable matrix pair,

$$
\delta(A, B)=\inf \left\{\|[E, F]\|:(A+E, B+F) \text { is not controllable, }[E, F] \in \mathbb{C}^{n \times(n+p)}\right\} .
$$

Here, $\|\cdot\|$ may denote any matrix norm. In this paper, we will use the spectral norm if not otherwise stated. The definition of $\delta(A, B)$ is motivated by the fact that modelling, discretization, approximation and other errors may have introduced uncertainties in the entries of the matrices $A$ and $B$. For example, a tiny value of $\delta(A, B)$ may signal that $\dot{x}=A x+B u$ is actually the approximation of an uncontrollable system. Moreover, $\delta(A, B)$ plays a prominent role in the sensitivity of various control problems, see $[5,8]$ and the references therein. Efficient algorithms for computing $\delta(A, B)$ can be found, e.g., in [6, 7].

In many applications, it is unreasonable to impose no restriction on the perturbation pair $(E, F)$ in (1). For example, if $A$ and $B$ are real matrices, it is natural to consider only real perturbations. In the most general setting, we consider an arbitrary perturbation class $\Delta \subseteq \mathbb{C}^{l \times r}$, structure matrices $L \in \mathbb{C}^{n \times l}, R \in \mathbb{C}^{r \times(n+p)}$, and define

$$
\delta_{\Delta}^{L, R}(A, B)=\inf \left\{\begin{array}{ll}
\|\Delta\|: \begin{array}{l}
(A+E, B+F) \text { is not controllable, } \\
{[E, F]=L \Delta R, \Delta \in \Delta .}
\end{array}
\end{array}\right\}
$$

\footnotetext{
*karow@math.tu-berlin.de, Institut für Mathematik, MA 4-5, TU Berlin, Str. des 17. Juni 136, D-10623 Berlin, Germany.

${ }^{\dagger}$ kressner@math.ethz.ch, Seminar for applied mathematics, ETH Zurich, Zurich, Switzerland. This author has been supported by a DFG Emmy Noether fellowship and in part by the Swedish Foundation for Strategic Research under the Frame Programme Grant A3 02:128.
} 
For brevity, we write $\delta_{\boldsymbol{\Delta}}(A, B)$ if $L=I_{n}, R=\operatorname{diag}\left(I_{n}, I_{p}\right)$, and $\delta_{\mathbb{R}}(A, B)$ if additionally $\boldsymbol{\Delta}=\mathbb{R}^{n \times n+p}$. We drop the subscript $\boldsymbol{\Delta}$ if $\boldsymbol{\Delta}=\mathbb{C}^{l \times r}$. Trivially, $\delta_{\boldsymbol{\Delta}}(A, B) \geq \delta(A, B)$. It may happen that $\delta(A, B)$ is tiny while $\delta_{\boldsymbol{\Delta}}(A, B) \gg 0$. In this case, $\delta(A, B)$ gives no indication on the (near) uncontrollability of $(A, B)$ if $[E, F]$ is restricted to be in $\Delta$. For example, consider the matrices

$$
A_{u}=\left[\begin{array}{cc}
0 & -u^{2} \\
1 & 0
\end{array}\right], \quad B=\left[\begin{array}{l}
1 \\
0
\end{array}\right],
$$

with the real parameter $u \geq 1$. Then $\delta\left(A_{u}, B\right) \leq 1 / u$ while $\delta_{\mathbb{R}}\left(A_{u}, B\right)=1$, see Appendix A for a proof.

The rest of this paper is organized as follows. In Section 2, we employ the notion of $\mu$-values to generalize Eising's [4] formula,

$$
\delta(A, B)=\inf _{\lambda \in \mathbb{C}} \sigma_{\min }([A-\lambda I, B]),
$$

to $\delta_{\boldsymbol{\Delta}}(A, B)$. (Note that $\sigma_{\mathrm{min}}$ denotes the minimal singular value of a matrix.) Deriving explicit expressions for $\delta_{\boldsymbol{\Delta}}(A, B)$ is, depending on the structure of $\boldsymbol{\Delta}$, a difficult task. Using results from $[1,15]$, we will first consider $\boldsymbol{\Delta}=\mathbb{R}^{n \times(n+p)}$. Already addressed by $\mathrm{Hu}$ and Davison [10], we point out that this case can also be directly obtained from [1, Theorem 3.1]. Demonstrating the flexibility gained by admitting the matrices $L$ and $R$ in the definition (2), it is shown to naturally include cases where either the state or input matrices are not perturbed. Section 3 provides computational formulas for structures $\boldsymbol{\Delta}$ that enforce some kind of symmetry in the perturbation of $A$. Extending results from [12], we cover Hermitian, symmetric and skew-symmetric perturbations. Finally, Section 4 reveals how the seemingly more general distance to uncontrollability for higher order systems fits into the developed framework.

\section{Structured distances and $\mu$-value}

We start our discussion of structured distances by extending Eising's formula (4) to admit factors $L$ and $R$.

Lemma 1 Let $L \in \mathbb{C}^{n \times l}, R \in \mathbb{C}^{r \times(n+p)}$ and $N \in \mathbb{C}^{n \times(n+p)}$. Suppose that $R$ has full column rank and $N$ has full row rank. Then

$$
\min \left\{\|\Delta\|: \Delta \in \mathbb{C}^{l \times r}, \operatorname{rank}(N-L \Delta R)<n\right\}=\left\|\left(N\left(R^{*} R\right)^{-1 / 2}\right)^{\dagger} L\right\|^{-1},
$$

where $\dagger$ denotes the Moore-Penrose inverse of a matrix.

Proof. Let $\Delta$ be such that $\operatorname{rank}(N-L \Delta R)<n$, which readily implies

$$
\operatorname{rank}\left(N\left(R^{*} R\right)^{-1 / 2}-L \Delta R\left(R^{*} R\right)^{-1 / 2}\right)<n .
$$

By an RQ decomposition, there is a unitary matrix $Q_{0} \in \mathbb{C}^{(n+m) \times(n+m)}$ such that

$$
N\left(R^{*} R\right)^{-1 / 2} Q_{0}^{*}=\left[N_{0}, 0\right]
$$

with $N_{0}$ invertible. We partition the matrix

$$
\Delta R\left(R^{*} R\right)^{-1 / 2} Q_{0}^{*}=\left[\Delta_{0}, \Delta_{1}\right]
$$


accordingly. Note that $R\left(R^{*} R\right)^{-1 / 2} Q_{0}^{*}$ has orthonormal columns and hence

$$
\widetilde{\Delta}:=\left[\Delta_{0}, 0\right] Q_{0}\left(R^{*} R\right)^{-1 / 2} R^{*}
$$

satisfies $\|\widetilde{\Delta}\|=\left\|\Delta_{0}\right\| \leq\|\Delta\|$. Moreover,

$$
n>\operatorname{rank}(N-L \Delta R)=\operatorname{rank}\left[N_{0}-L \Delta_{0},-L \Delta_{1}\right] \geq \operatorname{rank}\left[N_{0}-L \Delta_{0}, 0\right]=\operatorname{rank}(N-L \widetilde{\Delta} R),
$$

and thus we can always replace the matrix $\Delta$ in the minimization problem $(5)$ by $\widetilde{\Delta}$. The proof is completed by observing

$$
\begin{aligned}
\min \{\|\widetilde{\Delta}\|: \operatorname{rank}(N-L \widetilde{\Delta} R)<n\} & =\min \left\{\left\|\Delta_{0}\right\|: \operatorname{rank}\left(N_{0}-L \Delta_{0}\right)<n\right\}=\left\|N_{0}^{-1} L\right\|^{-1} \\
& =\left\|\left[N_{0}, 0\right]^{\dagger} L\right\|^{-1}=\left\|\left(N\left(R^{*} R\right)^{-1 / 2} Q_{0}^{*}\right)^{\dagger} L\right\|^{-1} \\
& =\left\|\left(N\left(R^{*} R\right)^{-1 / 2}\right)^{\dagger} L\right\|^{-1}
\end{aligned}
$$

$\square$

Corollary 1 Let $\boldsymbol{\Delta}=\mathbb{C}^{l \times r}$ and $(A, B) \in \mathbb{C}^{n \times n} \times \mathbb{C}^{n \times p}$ be controllable. Provided that $R$ has full column rank, the structured distance to uncontrollability defined in (2) satisfies

$$
\delta^{L, R}(A, B)=\left(\sup _{\lambda \in \mathbb{C}}\left\|\left(\left[A-\lambda I_{n}, B\right]\left(R^{*} R\right)^{-1 / 2}\right)^{\dagger} L\right\|\right)^{-1},
$$

Proof. Apply Lemma 1 to $N=\left[A-\lambda I_{n}, B\right]$.

In the following, we tacitly assume that $\Delta$ is a connected set containing the zero matrix. For $M \in \mathbb{C}^{n \times(n+p)}$ and $\boldsymbol{\Delta} \subseteq \mathbb{C}^{n \times(n+p)}$ we introduce a value closely related to the $\mu$-value [3] as follows

$$
\widetilde{\mu}_{\boldsymbol{\Delta}}(M)=\inf \{\|\Delta\|: \Delta \in \boldsymbol{\Delta}, \operatorname{rank}(M+\Delta)<n\} .
$$

The following result is a trivial consequence of this definition.

Lemma 2 Let $A \in \mathbb{C}^{n \times n}$ and $B \in \mathbb{C}^{n \times p}$ such that $(A, B)$ is controllable. Then the structured distance to uncontrollability satisfies

$$
\delta_{\boldsymbol{\Delta}}(A, B)=\inf _{\lambda \in \mathbb{C}} \widetilde{\mu}_{\boldsymbol{\Delta}}\left(\left[A-\lambda I_{n}, B\right]\right) .
$$

Note that for $\boldsymbol{\Delta}=\mathbb{C}^{n \times(n+p)}$ we have $\widetilde{\mu}_{\boldsymbol{\Delta}}(M)=\sigma_{\min }(M)$. Thus, equation (7) extends Eising's formula.

\subsection{Real perturbations}

For the important case of real perturbations, i.e. $\boldsymbol{\Delta}=\mathbb{R}^{l \times r}$ and $\boldsymbol{\Delta}=\mathbb{R}^{n \times(n+p)}$ respectively the following formula has been given in $[1,15]$ :

$$
\widetilde{\mu}_{\mathbb{R}}(M)=\sup _{\gamma \in(0,1]} \sigma_{2 n-1}\left(\left[\begin{array}{cc}
\Re M & -\gamma^{-1} \Im M \\
\gamma \Im M & \Re M
\end{array}\right]\right), \quad \text { for } M \in \mathbb{C}^{n \times(n+p)},
$$


where $\sigma_{2 n-1}$ denotes the second smallest singular value, and $\Re M$ and $\Im M$ denote the real and the imaginary part of $M$ respectively. Inserting the latter formula into (7) directly gives

$$
\delta_{\mathbb{R}}(A, B)=\inf _{\lambda_{R}, \lambda_{I} \in \mathbb{R}} \sup _{\gamma \in(0,1]} \sigma_{2 n-1}\left(\left[\begin{array}{cccc}
\Re A-\lambda_{R} I & \Re B & -\gamma^{-1}\left(\Im A-\lambda_{I} I\right) & -\gamma^{-1} \Im B \\
\gamma\left(\Im A-\lambda_{I} I\right) & \gamma \Im B & \Re A-\lambda_{R} I & \Re B
\end{array}\right]\right),
$$

see [10] for a different derivation of this formula. Example (3), see also Appendix A, shows that the ratio $\delta_{\mathbb{R}}(A, B) / \delta(A, B)$ can be arbitrarily large. The inner optimization in (9) involves a unimodal function and therefore can be conveniently addressed. In contrast, it is not clear how to perform the outer optimization other than by a brute-force grid search in $\mathbb{C}$. An optimization algorithm based on a completely different characterization of $\delta_{\mathbb{R}}(A, B)$ was developed by Wicks and DeCarlo [16]. However, as also pointed out in [10], this algorithm can become rather tedious for large $n$ and it is not clear whether it always attains the global minimum. Note that the definition of $\delta_{\mathbb{R}}(A, B)$ in [16] is not based on the spectral norm but on the Frobenius norm, which explains the different values of $\delta_{\mathbb{R}}(A, B)$ for an example reported in $[16$, Sec. VI] and [10, Sec. V]. The difference between spectral and Frobenius norms is due to the fact that the optimal perturbation in the spectral norm is usually of rank $2[1]$.

\subsection{Separable perturbations}

This section is concerned with the case that one of the matrices $A$ or $B$ is perturbed to a different extent. First of all, $R=\operatorname{diag}\left(\alpha I_{n}, \beta I_{m}\right)$ with $\alpha \neq 0$ and $\beta \neq 0$ allows to weight the perturbations $E$ and $F$ differently. For the unstructured case, we can apply Lemma 1 to obtain an expression for the corresponding distance to uncontrollability. Note, however, that the full rank assumption on $R$ in Lemma 1 excludes the cases $\alpha=0$ or $\beta=0$, i.e., when one of the coefficient matrices is not perturbed at all. These cases are treated in the following lemma.

\section{Lemma 3}

1. Let $\mathcal{E}=\left\{[E, 0]: E \in \mathbb{C}^{n \times n}\right\}$ and assume that $B^{*}$ has a nontrivial null space with orthonormal basis $U$. Then

$$
\delta_{\mathcal{E}}(A, B)=\min _{\lambda \in \mathbb{C}} \sigma_{\min }\left(U^{*}(A-\lambda I)\right) .
$$

2. Let $\mathcal{F}=\left\{[0, F]: F \in \mathbb{C}^{n \times m}\right\}$ and let the columns of $\operatorname{Eig}_{\lambda}(A)$ contain an orthonormal basis for the left eigenspace belonging to the eigenvalue $\lambda$ of $A$. Then

$$
\delta_{\mathcal{F}}(A, B)=\min _{\lambda \in \Lambda(A)} \sigma_{\min }\left(\operatorname{Eig}_{\lambda}(A)^{*} B\right)
$$

where $\Lambda(A)$ denotes the spectrum of $A$.

Proof. The matrix $[A-\lambda I+E, B]$ has rank smaller than $n$ if and only if there is a nonzero vector $x$ such that $x^{*}[A-\lambda I+E, B]=0$ or, equivalently, $x^{*}(A-\lambda I)=-x^{*} E$ under the condition $x^{*} B=0$. Note that

$$
\min \left\{\|E\|: x^{*}(A-\lambda I)=-x^{*} E\right\}=\left\|x^{*}(A-\lambda I)\right\| /\|x\| .
$$


see, e.g., [13]. Hence,

$$
\delta_{\mathcal{E}}(A, B)=\min _{\lambda \in \mathbb{C}} \min _{\substack{x \in \mathbb{C}^{n} \\ x^{*} B=0}} \frac{\left\|x^{*}(A-\lambda I)\right\|}{\|x\|}=\min _{\lambda \in \mathbb{C}} \min _{\|y\|=1}\left\|y^{*} U^{*}(A-\lambda I)\right\|,
$$

which shows Part 1. The proof of Part 2 is analogous, after observing that $\operatorname{rank}([A-\lambda I, B+$ $F])<n$ can only hold if $\lambda$ is an eigenvalue of $A$.

\section{Symmetry structures}

Recently, formulas for $\mu$-values with respect to Hermitian, symmetric, and skew-symmetric structures have been developed in [12]. In the following, we show how these results can be extended to obtain computable expressions for the structured controllability radius with respect to these perturbation classes.

\subsection{Hermitian matrices}

Lemma 4 Let $\boldsymbol{\Delta}=\left\{[E, F]: E \in \mathbb{C}^{n \times n}\right.$ is Hermitian and $\left.F \in \mathbb{C}^{n \times p}\right\}$. Then

$$
\delta_{\boldsymbol{\Delta}}(A, B)=\inf _{\lambda \in \mathbb{C}} \sqrt{\sup _{t \in \mathbb{R}} \lambda_{\min }\left(H_{0}(\lambda)+t H_{1}(\lambda)\right)},
$$

where $\lambda_{\min }(\cdot)$ denotes the smallest eigenvalue of a Hermitian matrix and

$$
H_{0}(\lambda)=(A-\lambda I)(A-\lambda I)^{*}+B B^{*}, \quad H_{1}(\lambda)=\frac{1}{2 \imath}\left(A-A^{*}\right)-\Im \lambda I .
$$

Proof. From Lemma 2 we have $\delta_{\boldsymbol{\Delta}}(A, B)=\inf _{\lambda \in \mathbb{C}} \widetilde{\mu}_{\boldsymbol{\Delta}}\left(\left[A-\lambda I_{n}, B\right]\right)$ where

$$
\begin{aligned}
\tilde{\mu}_{\boldsymbol{\Delta}}([\tilde{A}, B]) & =\inf _{[E, F] \in \boldsymbol{\Delta}}\{\|[E, F]\|: \operatorname{rank}([\tilde{A}+E, B+F])<n\} \\
& =\inf _{[E, F] \in \boldsymbol{\Delta}}\left\{\|[E, F]\|: \exists x \in \mathbb{C}^{n} \text { with }\|x\|=1, x^{*}[\tilde{A}+E, B+F]=0\right\} \\
& =\inf _{\|x\|=1[E, F] \in \boldsymbol{\Delta}}\left\{\|[E, F]\|: x^{*}[\tilde{A}+E, B+F]=0\right\} .
\end{aligned}
$$

The condition $x^{*}[\tilde{A}+E, B+F]=0$ implies $\left\|x^{*}[\tilde{A}, B]\right\|=\left\|x^{*}[E, F]\right\| \leq\|[E, F]\|$ and $\Im\left(x^{*} \tilde{A} x\right)=0$. In the following, we show that $E$ and $F$ can be chosen to attain the lower bound: $\left\|x^{*}[\tilde{A}, B]\right\|=\|[E, F]\|$. Since $\Im\left(x^{*} \tilde{A} x\right)=0$ there is a Hermitian matrix $E$ such that $x^{*} E=-x^{*} \tilde{A}$ and $\|E\|=\left\|x^{*} \tilde{A}\right\|$, see [13, Thm. 5.8]. Note that this relation also implies that $x$ is a left singular vector belonging to the largest singular value of $E$. Setting $F=x x^{*} B$ it holds that $\|F\|=\left\|x^{*} B\right\|$ and $x$ is a left singular vector belonging to the largest singular value of $F$. This implies that $x$ is also a left singular vector belonging to the largest singular value of $[E, F]$ and

$$
\|[E, F]\|=\left\|x^{*}[E, F]\right\|=\left\|x^{*}[\tilde{A}, B]\right\| .
$$

Consequently,

$$
\begin{aligned}
\tilde{\mu}_{\boldsymbol{\Delta}}([A-\lambda I, B]) & =\inf _{\|x\|=1}\left\{\left\|x^{*}[A-\lambda I, B]\right\|: \Im\left(x^{*}(A-\lambda I) x\right)=0\right\} \\
& =\sqrt{\inf _{\|x\|=1}\left\{x^{*} H_{0}(\lambda) x: x^{*} H_{1}(\lambda) x=0\right\}} .
\end{aligned}
$$


By a result in [12], the latter minimization problem can be written as

$$
\widetilde{\mu}_{\boldsymbol{\Delta}}([A-\lambda I, B])=\sqrt{\sup _{t \in \mathbb{R}} \lambda_{\min }\left(H_{0}(\lambda)+t H_{1}(\lambda)\right)},
$$

which concludes the proof.

Let us briefly consider the case that $A$ itself is Hermitian. Then the matrix $H_{1}(\lambda)$ in Lemma 4 becomes positive definite for $\lambda \notin \mathbb{R}$, in which case the supremum is infinite. Hence, we can restrict the outer optimization in (10) to $\lambda \in \mathbb{R}$ yielding

$$
\delta_{\boldsymbol{\Delta}}(A, B)=\inf _{\lambda \in \mathbb{R}} \sigma_{\min }([A-\lambda I, B])
$$

for Hermitian $A$.

For non-Hermitian $A$, the range of the outer optimization in (10) can be restricted to all $\lambda$ satisfying $\Im \lambda \in\left[-\lambda_{\max }\left(\frac{A-A^{*}}{2 \imath}\right),-\lambda_{\min }\left(\frac{A-A^{*}}{2 \imath}\right)\right]$. See [12] for restricting the range of the inner optimization, which involves a unimodal function and is thus considerably simple.

\subsection{Symmetric matrices}

The next lemma is concerned with complex symmetric perturbations. Note that $E^{\top}$ denotes the complex transpose of $E$.

Lemma 5 Let $\boldsymbol{\Delta}=\left\{[E, F]: E \in \mathbb{C}^{n \times n}\right.$ satisfies $\left.E=E^{\top}, F \in \mathbb{C}^{n \times p}\right\}$. Then $\delta_{\boldsymbol{\Delta}}(A, B)=$ $\delta(A, B)$.

Proof. The lemma can be proven along the lines of the proof of Lemma 4. However, in contrast to the Hermitian case there always exists a complex symmetric matrix $E$ such that $x^{*} E=-x^{*} \tilde{A}$ and $\|E\|=\left\|x^{*} \tilde{A}\right\|$, see [13, Thm. 5.8]. Thus the additional constraint in (11) can be dropped, which gives

$$
\widetilde{\mu}_{\boldsymbol{\Delta}}([A-\lambda I, B])=\inf _{\|x\|=1}\left\|x^{*}[A-\lambda I, B]\right\|=\sigma_{\min }([A-\lambda I, B])
$$

and therefore completes the proof.

\subsection{Skew-symmetric matrices}

Lemma 6 Let $\boldsymbol{\Delta}=\left\{[E, F]: E \in \mathbb{C}^{n \times n}\right.$ satisfies $\left.E=-E^{\top}, F \in \mathbb{C}^{n \times p}\right\}$. Then

$$
\delta_{\boldsymbol{\Delta}}(A, B)=\inf _{\lambda \in \mathbb{C}} \sqrt{\sup _{t \geq 0} \lambda_{2 n-1}\left(\left[\begin{array}{cc}
H(\lambda) & t \overline{S(\lambda)} \\
t S(\lambda) & \overline{H(\lambda)}
\end{array}\right]\right)}
$$

where $\lambda_{2 n-1}(\cdot)$ denotes the second smallest eigenvalue of a $2 n \times 2 n$ Hermitian matrix and

$$
H(\lambda)=(A-\lambda I)(A-\lambda I)^{*}+B B^{*}, \quad S(\lambda)=\frac{1}{2}\left(A+A^{\top}\right)-\lambda I .
$$

Proof. Proceeding as in the proof of Lemma 4, we obtain

$$
\widetilde{\mu}_{\boldsymbol{\Delta}}([\tilde{A}, B])=\inf _{\|x\|=1} \inf _{[E, F] \in \boldsymbol{\Delta}}\left\{\|[E, F]\|: x^{*}[\tilde{A}+E, B+F]=0\right\} .
$$


From the skew-symmetry of $E$ it follows that $x^{*} E=-x^{*} \tilde{A}$ implies $x^{\top} \tilde{A} x=0$. On the other hand, provided that $x^{\top} \tilde{A} x=0$ is satisfied, we can always find a skew-symmetric matrix $E$ such that $x^{*} E=-x^{*} \tilde{A}$ and $\|E\|=\left\|x^{*} \tilde{A}\right\|$. Once again, this is a consequence of Theorem 5.8 in [13]. Similarly to (11), one can show that

$$
\begin{aligned}
\widetilde{\mu}_{\Delta}([A-\lambda I, B]) & =\inf _{\|x\|=1}\left\{\left\|x^{*}[A-\lambda I, B]\right\|: x^{\top}(A-\lambda I) x=0\right\} \\
& =\sqrt{\inf _{\|x\|=1}\left\{x^{*} H(\lambda) x: x^{\top} S(\lambda) x=0\right\}} .
\end{aligned}
$$

The proof is completed by applying results from [12] to the latter optimization problem.

\subsection{Extension to related structures}

Let $J \in \mathbb{C}^{n \times n}$ be a fixed unitary matrix and define the structures

$$
\boldsymbol{\Delta}=\left\{[E, F]: E \in \mathbb{S}, F \in \mathbb{C}^{n \times p}\right\}, \quad \boldsymbol{\Delta}_{J}=\left\{[E, F]: J E \in \mathbb{S}, F \in \mathbb{C}^{n \times p}\right\},
$$

for some $\mathbb{S} \subseteq \mathbb{C}^{n \times n}$. Then, directly by the definition of $\delta_{\boldsymbol{\Delta}}(A, B)$,

$$
\widetilde{\mu}_{\Delta_{J}}([A-\lambda I, B])=\widetilde{\mu}_{\Delta}([J A-\lambda J, J B]) .
$$

This relation allows to extend the results for $\delta_{\boldsymbol{\Delta}}(A, B)$ of Lemmas 4,5 , and 6 to derive similar formulas for the structured distance $\delta_{\boldsymbol{\Delta}_{J}}(A, B)$, provided of course that $\boldsymbol{\Delta}$ corresponds to the set of Hermitian, symmetric, or skew-symmetric matrices.

To illustrate this, let us consider the set of Hamiltonian matrices for which $\mathbb{S}$ is the set of Hermitian matrices and $J=\left[\begin{array}{cc}0 & I \\ -I & 0\end{array}\right]$. Inserting (13) in (11) leads to

$$
\delta_{\boldsymbol{\Delta}_{J}}(A, B)=\inf _{\lambda \in \mathbb{C}} \sqrt{\sup _{t \in \mathbb{R}} \lambda_{\min }\left(H_{0, J}(\lambda)+t H_{1, J}(\lambda)\right)},
$$

where

$$
H_{0, J}(\lambda)=J\left((A-\lambda I)(A-\lambda I)^{*}+B B^{*}\right) J^{*}, \quad H_{1, J}(\lambda)=\frac{1}{2 \imath}\left(J A-(J A)^{*}\right)+2 \imath \Re \lambda J .
$$

\section{Higher order systems}

Let us consider a higher order linear time-invariant system

$$
A_{k} x^{(k)}(t)+A_{k-1} x^{(k-1)}(t)+\cdots+A_{1} x^{\prime}(t)+A_{0} x(t)=B u(t)
$$

where $A_{0}, \ldots, A_{k} \in \mathbb{C}^{n \times n}$ and $B \in \mathbb{C}^{n \times m}$. Furthermore, we assume that $A_{k}$ is nonsingular. Then (14) is controllable if and only if $\operatorname{rank}[P(\lambda), B]=n$ for all $\lambda \in \mathbb{C}$, with the matrix polynomial

$$
P(\lambda)=A_{k} \lambda^{k}+A_{k-1} \lambda^{k-1}+\cdots+A_{1} \lambda+A_{0} .
$$

see $[9,14]$. The polynomial corresponding to a perturbation of $(14)$ is denoted by

$$
P_{E}(\lambda)=\alpha_{k} E_{k} \lambda^{k}+\alpha_{k-1} E_{k-1} \lambda^{k-1}+\cdots+\alpha_{1} E_{1} \lambda+\alpha_{0} E_{0}
$$


for fixed nonnegative scalars $\alpha_{0}, \ldots, \alpha_{k}$. These scalars account for the fact that some coefficients of (14) may be less (or not at all) affected by perturbations. The distance to uncontrollability of (14) can then be defined as

$$
\delta(P, B)=\inf \left\{\left\|\left[E_{0}, \ldots, E_{k}, F\right]\right\|:\left(P+P_{E}, B+F\right) \text { is not controllable. }\right\}
$$

To avoid technical difficulties, we tacitly assume that the perturbed leading factor $A_{k}+E_{k}$ always remains nonsingular (see [2] for handling singular leading factors in the case $k=1$ ). Note that we can write $P_{E}(\lambda)=\Delta R$, where

$$
R=\left[\begin{array}{cc}
\alpha_{0} & 0 \\
\alpha_{1} \lambda & 0 \\
\vdots & \vdots \\
\alpha_{k} \lambda^{k} & 0 \\
0 & 1
\end{array}\right] .
$$

Thus,

$$
\delta_{\Delta}(P, B)=\inf \left\{\|\Delta\|: \Delta \in \mathbb{C}^{n \times(k n+m)}, \operatorname{rank}([P(\lambda), B]+\Delta R)<n \text { for some } \lambda\right\} .
$$

Using that

$$
R^{*} R=\left[\begin{array}{cc}
s(\lambda) & 0 \\
0 & 1
\end{array}\right], \quad s(\lambda):=\sum_{j=0}^{k} \alpha_{j}^{2}|\lambda|^{2 j},
$$

Lemma 1 applied to (17) yields

$$
\begin{aligned}
\delta_{\boldsymbol{\Delta}}(P, B) & =\left(\sup _{\lambda \in \mathbb{C}}\left\|\left([P(\lambda), B]\left(R^{*} R\right)^{-1 / 2}\right)^{\dagger}\right\|\right)^{-1} \\
& =\left(\sup _{\lambda \in \mathbb{C}}\left\|([P(\lambda) / \sqrt{s(\lambda)}, B])^{\dagger}\right\|\right)^{-1} \\
& =\inf _{\lambda \in \mathbb{C}} \sigma_{\min }([P(\lambda) / \sqrt{s(\lambda)}, B]) .
\end{aligned}
$$

Note that this result is also covered by Theorem 2.3 in [14]. However, we feel that our approach is conceptually simpler.

\section{Conclusions}

We have derived characterizations of the structured distance to uncontrollability for several practically relevant structures. The obtained expressions can be computed numerically by performing a grid search in the complex plane and evaluating a certain function at each grid value. This inner function evaluation could be addressed by a general optimization procedure presented in [17]. However, this procedure may miss global minima and is thus not entirely reliable. In contrast, the expressions for symmetry structures presented in this paper only require the minimization of a unimodal function at each grid point, which is both inexpensive and reliable.

Note that recently developed algorithms $[6,7]$ for computing the unstructured distance to uncontrollability do not require any form of grid search. This is achieved by specifically 
exploiting the fact that the inner function in the unstructured case is the minimum singular value of a matrix. It is currently under investigation how these ideas can be extended to structured distances.

Finally, we note that the results of this paper can be extended to the stabilizability radius by restricting the set of admissible $\lambda$ to $\mathbb{C}^{+}$.

\section{Acknowledgments}

We are grateful to Emre Mengi for pointing out that a tempting extension of Lemma 1 to arbitrary matrix structures does not hold.

\section{A Appendix}

The proposition below gives a simple example for the fact that the ratio between the real and the complex distance to uncontrollability can be arbitrarily large.

Proposition 7 Let $u \geq 1, A_{u}=\left[\begin{array}{cc}0 & -u^{2} \\ 1 & 0\end{array}\right], b_{0}=\left[\begin{array}{l}1 \\ 0\end{array}\right]$. Then

$$
\begin{array}{r}
\delta_{\mathbb{C}}\left(A_{u}, b_{0}\right) \leq 1 / u, \\
\delta_{\mathbb{R}}\left(A_{u}, b_{0}\right)=1,
\end{array}
$$

and hence

$$
\lim _{u \rightarrow \infty} \frac{\delta_{\mathbb{R}}\left(A_{u}, b_{0}\right)}{\delta_{\mathbb{C}}\left(A_{u}, b_{0}\right)}=\infty
$$

The proof of the proposition is based on the following lemma on 2-dimensional systems.

Lemma 8 Let $A \in \mathbb{C}^{2 \times 2}, b \in \mathbb{C}^{2 \times 1}$. Then the following holds.

(i) The pair $(A, b)$ is not controllable if and only if either $b=0$ or $b$ is an eigenvector of $A$.

(ii) If $A$ and $b$ are real then the real distance of $(A, b)$ to uncontrollability satisfies

$$
\delta_{\mathbb{R}}(A, b) \geq \min \left\{\|b\|, \min _{\lambda \in \mathbb{R}} \sigma_{2}\left(A-\lambda I_{2}\right)\right\} .
$$

Proof. ( $i)$ The pair $(A, b)$ is not controllable iff the vectors $b$ and $A b$ are linearly dependent 1 , i.e. iff either $b=0$ or $b \neq 0$ and $0=A b-\lambda b=\left(A-\lambda I_{2}\right) b$ for some $\lambda \in \mathbb{C}$.

(ii) Let $(E, f) \in \mathbb{R}^{2 \times 2} \times \mathbb{R}^{2 \times 1}$. Suppose $(A+E, b+f)$ is not controllable. Then by $(i)$ either $f=-b$ or $f \neq-b$ and $\left(A+E-\lambda I_{2}\right)(b+f)=0$ for some $\lambda \in \mathbb{R}$. In the first case, $\|[E, f]\| \geq\|[0, f]\|=\|b\|$. In the second case, $\|b+f\| \neq 0$ and

$$
\|E(b+f)\|=\left\|\left(A-\lambda I_{2}\right)(b+f)\right\| \geq \sigma_{2}\left(A-\lambda I_{2}\right)\|b+f\| .
$$

This implies $\|[E, f]\| \geq\|E\| \geq\|E(b+f)\| /\|b+f\| \geq \sigma_{2}\left(A-\lambda I_{2}\right)$.

We are now in a position to prove Propositon 7. Let $E=0 \in \mathbb{C}^{2 \times 2}$ and $f=\left[\begin{array}{ll}0 & 1 /(i u)\end{array}\right]^{T}$. Then $\left(A_{u}+E\right)\left(b_{0}+f\right)=(i u)\left(b_{0}+f\right)$. Hence, $\left(A_{u}+E, b_{0}+f\right)$ is uncontrollable by the first

\footnotetext{
${ }^{1}$ This is immediate from the following well known controllability criterion: The pair $(A, B) \in \mathbb{C}^{n \times n} \times \mathbb{C}^{n \times p}$ is controllable if and only if $\operatorname{rank}\left[B, A B, \ldots, A^{n-1} B\right]=n$.
} 
statement of the lemma. Thus $\delta_{\mathbb{C}}\left(A_{u}, b_{0}\right) \leq\|[E, f]\|=1 / u$. Now, let $E=\left[\begin{array}{cc}0 & 0 \\ -1 & 0\end{array}\right], f=0$. Then $\left(A_{u}+E\right)\left(b_{0}+f\right)=0$, and there hence $\delta_{\mathbb{R}}\left(A_{u}, b_{0}\right) \leq\|[E, f]\|=1$. It remains to show $\delta_{\mathbb{R}}\left(A_{u}, b_{0}\right) \geq 1$. To this end we consider the matrix

$$
\begin{aligned}
P_{u, \lambda} & =\left(A_{u}-\lambda I_{2}\right)^{T}\left(A_{u}-\lambda I_{2}\right)-I_{2} \\
& =\left[\begin{array}{cc}
\lambda^{2} & \lambda\left(1-u^{2}\right) \\
\lambda\left(1-u^{2}\right) & u^{4}+\lambda^{2}-1
\end{array}\right] .
\end{aligned}
$$

For all $\lambda \in \mathbb{R}, u \geq 1, P_{u, \lambda}$ has nonnegative diagonal elements, and

$$
\operatorname{det}\left(P_{u, \lambda}\right)=\lambda^{2}\left(\lambda^{2}+2\left(u^{2}-1\right)\right) \geq 0 .
$$

Hence, $P_{u, \lambda}$ is positive semidefinite. Thus $\sigma_{2}\left(A_{u}-\lambda I_{2}\right) \geq 1$ for $\lambda \in \mathbb{R}, u \geq 1$. Now, the second statement of the lemma yields

$$
\delta_{\mathbb{R}}\left(A_{u}, b_{0}\right) \geq \min \left\{\left\|b_{0}\right\|, \min _{\lambda \in \mathbb{R}} \sigma_{2}\left(A_{u}-\lambda I_{2}\right)\right\} \geq 1 .
$$

\section{References}

[1] B. Bernhardsson, A. Rantzer, and L. Qiu. Real perturbation values and real quadratic forms in a complex vector space. Linear Algebra Appl., 270:131-154, 1998.

[2] R. Byers. The descriptor controllability radius. In U. Helmke, R. Mennicken, and J. Saurer, editors, Proceedings of the Conference on the Mathematical Th eory of Networks and Systems, MTNS '93, pages 85-88. Akademie Verlag, Berlin, 1994.

[3] J. C. Doyle. Analysis of feedback systems with structured uncertainties. IEE Proc., 129(6), 1982.

[4] R. Eising. Between controllable and uncontrollable. Systems Control Lett., 4(5):263-264, 1984.

[5] P. Gahinet and A. J. Laub. Algebraic Riccati equations and the distance to the nearest uncontrollable pair. SIAM J. Control Optim., 30(4):765-786, 1992.

[6] M. Gu. New methods for estimating the distance to uncontrollability. SIAM J. Matrix Anal. Appl., 21(3):989-1003, 2000.

[7] M. Gu, E. Mengi, M.L. Overton, J. Xia, and J. Zhu. Fast methods for estimating the distance to uncontrollability. SIAM J. Matrix Anal. Appl., 28(2):477-502, 2006.

[8] N. J. Higham, M. Konstantinov, V. Mehrmann, and P. Petkov. The sensitivity of computational control problems. IEEE Control Systems Magazine, 24(1):28-43, 2004.

[9] N. J. Higham and F. Tisseur. More on pseudospectra for polynomial eigenvalue problems and applications in control theory. Linear Algebra Appl., 351/352:435-453, 2002. 
[10] G. Hu and E. J. Davison. Real controllability/stabilizability radius of LTI systems. IEEE Trans. Automat. Control, 49(2):254-257, 2004.

[11] R. E. Kalman. Mathematical description of linear systems. SIAM J. Control Optim., 1:152-192, 1963.

[12] M. Karow. $\mu$-values and spectral value sets for linear perturbation classes defined by a scalar product. Matheon-preprint 406. Available from http://www.matheon.de/ research/list_preprints.asp. Submitted, 2007.

[13] D. S. Mackey, N. Mackey, and F. Tisseur. Structured mapping problems for matrices associated with scalar products part I: Lie and Jordan algebras. SIAM J. of Matrix Analysis and Appl., 29(4):1389-1410, 2007.

[14] E. Mengi. On the estimation of the distance to uncontrollability for higher order systems. SIAM J. of Matrix Analysis and Appl., 30(1):154-172, 2008.

[15] L. Qiu, B. Bernhardsson, A. Rantzer, E. J. Davison, P. M. Young, and J. C. Doyle. A formula for computation of the real stability radius. Automatica J. IFAC, 31(6):879-890, 1995.

[16] M. Wicks and R. A. DeCarlo. Computing the distance to an uncontrollable system. IEEE Trans. Automat. Control, 36(1):39-49, 1991.

[17] M. A. Wicks and R. A. DeCarlo. Computing most nearly rank-reducing structured matrix perturbations. SIAM J. Matrix Anal. Appl., 16(1):123-137, 1995. 